\title{
Properties of dust at the Galactic center probed by AKARI far-infrared spectral mapping
}

\section{Detection of a dust feature}

\author{
H. Kaneda ${ }^{1}$, A. Yasuda ${ }^{1,2}$, T. Onaka ${ }^{3}$, M. Kawada ${ }^{2}$, N. Murakami ${ }^{6}$, T. Nakagawa ${ }^{2}$, Y. Okada ${ }^{4}$, and H. Takahashi ${ }^{5}$ \\ ${ }^{1}$ Graduate School of Science, Nagoya University, Chikusa-ku, 464-8602 Nagoya, Japan \\ e-mail: kaneda@u.phys.nagoya-u.ac.jp \\ 2 Institute of Space and Astronautical Science, Japan Aerospace Exploration Agency, Sagamihara, 252-5210 Kanagawa, Japan \\ 3 Department of Astronomy, Graduate School of Science, University of Tokyo, Bunkyo-ku, 113-0033 Tokyo, Japan \\ ${ }^{4}$ I. Physikalisches Institut, Universität zu Köln, Zülpicher Str. 77, 50937 Köln, Germany \\ 5 Institute of Astronomy, Graduate School of Science, University of Tokyo, Mitaka, 181-0015 Tokyo, Japan \\ ${ }^{6}$ Bisei Astronomical Observatory, Ibara, 714-1411 Okayama, Japan
}

Received 17 March 2012 / Accepted 12 May 2012

\begin{abstract}
Aims. We investigate the properties of interstellar dust in the Galactic center region toward the Arches and Quintuplet clusters. Methods. With the Fourier Transform Spectrometer of the AKARI/Far-Infrared Surveyor, we performed the far-infrared $\left(60-140 \mathrm{~cm}^{-1}\right)$ spectral mapping of an area of about $10^{\prime} \times 10^{\prime}$ that includes both clusters to obtain a low-resolution $\left(R=1.2 \mathrm{~cm}^{-1}\right)$ spectrum at every spatial bin of $30^{\prime \prime} \times 30^{\prime \prime}$.

Results. We derive the spatial variations in the dust continuum emission at different wavenumbers, which are compared with those of the [OIII] $88 \mu \mathrm{m}\left(113 \mathrm{~cm}^{-1}\right)$ emission and the $\mathrm{OH} 119 \mu \mathrm{m}\left(84 \mathrm{~cm}^{-1}\right)$ absorption. The spectral fitting shows that two modified blackbody components with temperatures of $\sim 20 \mathrm{~K}$ and $\sim 50 \mathrm{~K}$ can reproduce most of the continuum spectra. For some spectra, however, we find that there is a significant excess on top of a modified blackbody continuum around $80-90 \mathrm{~cm}^{-1}(110-130 \mu \mathrm{m})$.

Conclusions. The warmer dust component is spatially correlated with the [OIII] emission and hence likely to be associated with the highly-ionized gas locally heated by intense radiation from the two clusters. The excess emission probably represents a dust feature, which is found to be spatially correlated with the $\mathrm{OH}$ absorption and a $\mathrm{CO}$ cloud. We find that a dust model including micron-sized graphite grains can quite closely reproduce the observed spectrum with the dust feature.
\end{abstract}

Key words. dust, extinction - ISM: clouds - Galaxy: center - infrared: ISM

\section{Introduction}

The center of our Galaxy exhibits very complicated structures in the interstellar medium (ISM) on spatial scales between $\sim 1 \mathrm{pc}$ and $\sim 100 \mathrm{pc}$, which harbors several active star formation sites (e.g., Yusef-Zadeh et al. 2009). For example, the most massive young stellar clusters in our Galaxy, namely the Arches and the Quintuplet cluster (Cotera et al. 1992; Nagata et al. 1995; Serabyn et al. 1998; Figer et al. 1999), are located near the Galactic center, which are thought to be responsible for the ionization of the two prominent filamentary structures, the Arched Filaments (Cotera et al. 1996; Lang et al. 2001) and the Sickle (Simpson et al. 1997; Figer et al. 1999; Rodríguez-Fernández et al. 2001), respectively. Intense stellar winds and ultraviolet (UV) radiation from these massive clusters are likely to have major impacts on their ambient ISM (Lang et al. 2005). However, the structures are so complicated that the clouds associated with and influenced by these clusters are rather difficult to clearly resolve.

In the far-infrared (far-IR), the ISM in the Galactic center region has been studied by both gas forbidden line and dust continuum emission. Genzel et al. (1990) observed the strong far-IR fine-structure [CII] line at the Radio Arc and the $+20 /+50 \mathrm{~km} \mathrm{~s}^{-1}$ molecular clouds with the Kuiper Airborne Observatory, who suggested that the $[\mathrm{CII}]$ emission comes from interfaces between the molecular clouds and ionized zones. The Infrared Space Observatory (ISO) detected various far-IR emission lines, which were interpreted as the result of excitation by the UV radiation produced by the Arches and the Quintuplet cluster (Rodríguez-Fernández et al. 2001). Cotera et al. (2005), Simpson et al. (2007), and Yasuda et al. (2009) showed that the ratio of the highly-ionized to the lowly-ionized line intensity decreases with the distance from the Arches cluster with ISO, Spitzer, and AKARI, respectively, which indicates that the Arched Filaments are ionized by the Arches cluster.

As for early studies of the dust emission at the Galactic center, for example, Cox \& Laureijs (1989) estimated the average dust temperature to be $27 \mathrm{~K}$ from the far-IR observations of a central $450 \times 300 \mathrm{pc}$ region with IRAS. Lis et al. (2001) derived the dust temperature of $18 \mathrm{~K}$ for the cold giant molecular cloud at Galactic longitudes of between $\ell \sim 0.2^{\circ}$ and $0.6^{\circ}$ and latitudes $b \sim \pm 0.1^{\circ}$ with ISO; they found that the contribution of warm dust $(\sim 35 \mathrm{~K})$ was very small. Rodríguez-Fernández et al. (2004) observed the 18 molecular clouds located far from thermal radio-continuum sources in the Galactic center, showing that the dust continuum emission could be modeled with two temperatures: a cold component with temperature of $\sim 15 \mathrm{~K}$ and a warm component with temperatures varying between $25 \mathrm{~K}$ and $40 \mathrm{~K}$ from source to source. Etxaluze et al. (2011) analyzed the Herschel and ISO data of the Galactic center region, who 
found that the spectral energy distribution (SED) of central $2 \mathrm{pc}$ in Sagittarius A* (Sgr A*) could be fitted by a three-temperature modified blackbody curve with temperatures of 90,45 , and $23 \mathrm{~K}$. Fitting a single-temperature modified blackbody curve to the PACS $70 \mu \mathrm{m}$ and SPIRE 250, 350, and $500 \mu \mathrm{m}$ data, they found that the dust temperatures of the Arched Filaments, the Sickle, and the Radio Arc are $\sim 35 \mathrm{~K}, \sim 35 \mathrm{~K}$, and $\sim 28 \mathrm{~K}$, respectively, while those for most other regions around the Galactic center are cooler than $\sim 25 \mathrm{~K}$. Hence, these past studies consistently revealed that warm dust is a relatively minor constituent of the far-IR continuum emission, despite the presence of the very active star formation sites. Nakagawa et al. (1998) found that the Galactic center shows a global depression in the [CII]/far-IR ratio along the Galactic plane; with the help of ISO [OI] data, Yasuda et al. (2008) concluded that the relatively strong far-IR continuum emission toward the Galactic center is caused mostly by non-C-ionizing soft radiation from old stellar populations.

In the present paper, we report our results of the far-IR spectral mapping of the Arches and Quintuplet cluster region near the Galactic center, using the Fourier Transform Spectrometer (FTS) of the Far-IR Surveyor (FIS) onboard AKARI (Murakami et al. 2007; Kawada et al. 2007, 2008). The AKARI FIS-FTS instrument covers the wavenumber range of $60-140 \mathrm{~cm}^{-1}$ with two kinds of two-dimensional detector arrays, thereby we simultaneously obtained the line and continuum maps of ionized sources and clouds around the clusters in spatially and spectrally continuous ways, which contain important information about the ISM in the Galactic center region. Early results for the line emission maps of the same region were reported in Yasuda et al. (2009). In this paper, we present the spatial variations in the spectral shapes by focusing mainly on dust continuum emission. We report not only our results for the dust clouds associated with and influenced by the Arches and Quintuplet clusters, which had been the original purpose of this study, but also for a dust feature, which is unexpectedly detected in spectra mainly toward the Arches cluster region.

\section{Observation and data reduction}

The observations were carried out by five pointed observations in September 2006 and March 2007. AKARI FIS-FTS has two kinds of detector arrays (WIDE-S and WIDE-L; hereafter SW and $\mathrm{LW}$, respectively), and we obtain the spectra of the SW $\left(88-140 \mathrm{~cm}^{-1}, 71-114 \mu \mathrm{m}\right)$ and the LW range $\left(60-88 \mathrm{~cm}^{-1}\right.$, 114-167 $\mu \mathrm{m})$, simultaneously. The effective angular resolution is $39^{\prime \prime}-44^{\prime \prime}$ for SW and 53"-57" for LW (Kawada et al. 2008). The full-resolution mode was used with a very short reset interval of $0.1 \mathrm{~s}$. Figure 1 shows the areas mapped with the SW and LW arrays, where the radio $20 \mathrm{~cm}$ continuum map (Yusef-Zadeh et al. 1984) is superimposed. The difference in the spatial coverage between the SW and the LW map is due to the difference in the fields of view of the SW and LW array detectors (Kawada et al. 2008). As can be seen in the figure, both SW and LW cover the Arches and Quintuplet clusters, together with the Arched Filaments and the Sickle.

We used the official pipeline for our data analyses. Details of the data reduction processes and calibration were described in Murakami et al. (2010). Although the observational data were obtained in the full-resolution mode $\left(R=0.19 \mathrm{~cm}^{-1}\right)$, we used an SED mode $\left(R=1.2 \mathrm{~cm}^{-1}\right)$ analysis to increase the signal-tonoise ratios of the continuum emission, because the main purpose of this paper is to study dust emission. We used both of the short sides of the FTS interferogram with respect to the center burst for the SED analysis rather than one long side for the
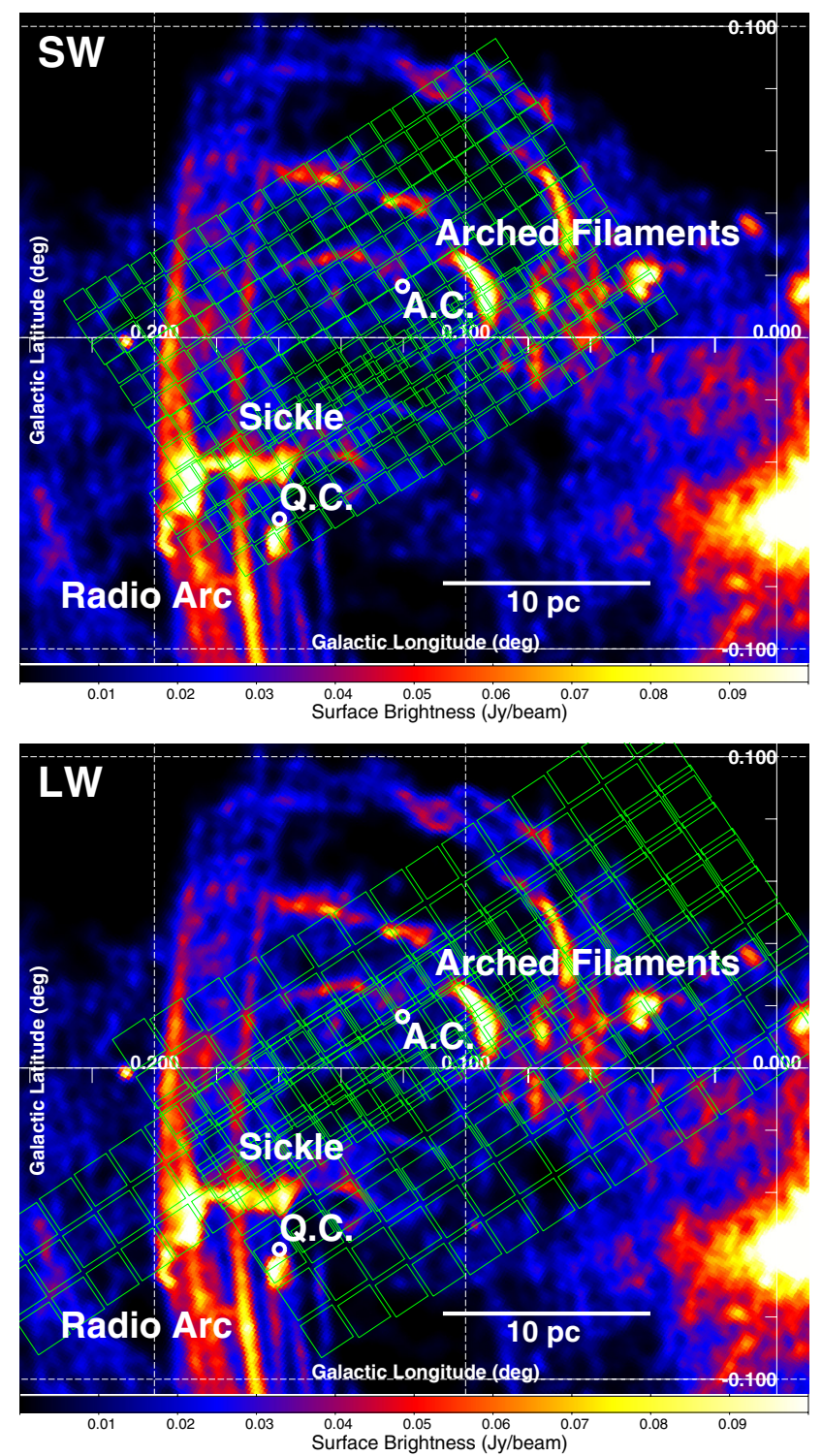

Fig. 1. Areas mapped with the SW (top) and the LW (bottom) array, both overlaid on the radio $20 \mathrm{~cm}$ continuum map (Yusef-Zadeh et al. 1984). The positions of the Arches and Quintuplet clusters (here and hereafter, A.C. and Q.C. in the figures), together with the names of the primary structures, are added to the panels. The field of view of each pixel is shown in the small box.

full-resolution analysis; owing to the limitation in the movement of the FTS scanning mirror, only one side is set to be long for the optical path difference in the full-resolution mode (Kawada et al. 2008). The usage of both sides increases the tolerance to the effects of detector artifacts such as transient response. Another merit of the SED mode analysis is that it produces spectra that are much less affected by the channel fringes than the full-resolution mode analysis.

We note that the pixels of the SW and LW detectors do not observe the same area of sky. Therefore, we first created a map for each spectral bin of $0.6 \mathrm{~cm}^{-1}$ for both the SW and LW detectors and then regridded it with the common spatial bin of $30^{\prime \prime} \times 30^{\prime \prime}$. At this stage, we derived 122 maps with a spectral bin of $0.6 \mathrm{~cm}^{-1}$ to create a spectrum at every spatial bin of $30^{\prime \prime} \times 30^{\prime \prime}$ for the region overlapping between the SW and LW maps. Using fluxes in two contiguous spectral bins and eight contiguous spatial pixels, we then averaged $27(=3 \times 9)$ fluxes in total to obtain 

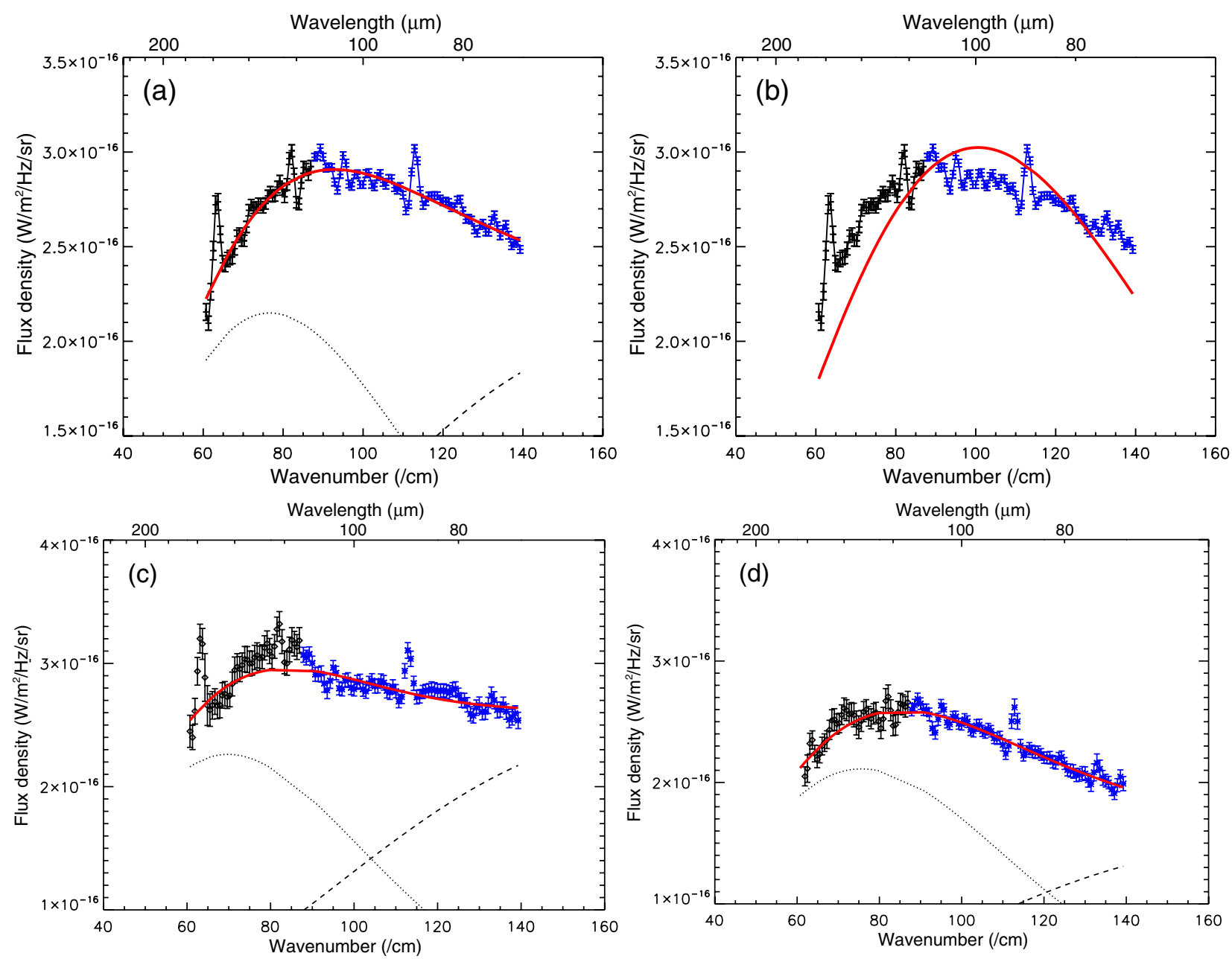

Fig. 2. AKARI/FIS-FTS spectra of a), b) the total region covered by both SW and LW, c) the regions toward the Arches cluster, and d) the Quintuplet cluster. The results of the double-temperature (panels a), c), d)) and single-temperature (panel b)) modified blackbody model fitting are shown together, where the wavenumber ranges corresponding to the emission lines ([OIII]: 111 to $115 \mathrm{~cm}^{-1}$, [NII]: 81 to $85 \mathrm{~cm}^{-1}$, [CII]: 62 to $\left.66 \mathrm{~cm}^{-1}\right)$ and the excess feature $\left(80\right.$ to $\left.90 \mathrm{~cm}^{-1}\right)$ are masked in the spectral fitting. Here and hereafter, the different colors are used to show the spectra derived from the SW (blue) and LW (black) detectors. The aperture regions used to extract spectra (c) and (d) are indicated in Fig. 4a.

a local spectrum for every spectral bin. Finally, the spectral mismatches, caused mainly by the difference in the field-of-view and also possibly by residual calibration uncertainties, were removed by scaling the LW to match the SW spectra at several overlapping and adjacent spectral bins by factors of $0.85-1.11$.

Figure $2 \mathrm{a}$ shows the spectrum integrated over the total area covered by both SW and LW (Fig. 1). The SW and LW spectra were combined to form one single spectrum as described above. From the total spectrum and local spectra, we detected the three far-IR fine-structure lines, [OIII] $88 \mu \mathrm{m}\left(113 \mathrm{~cm}^{-1}\right)$, [NII] $122 \mu \mathrm{m}\left(82 \mathrm{~cm}^{-1}\right)$, and [CII] $158 \mu \mathrm{m}\left(63 \mathrm{~cm}^{-1}\right)$, as well as the $\mathrm{OH}$ absorption at $119 \mu \mathrm{m}\left(84 \mathrm{~cm}^{-1}\right)$ due to the fundamental rotational mode, and dust continuum emission over the whole wavenumber range of FIS-FTS.

\section{Results}

\subsection{Dust continuum maps}

We derived continuum maps, using the local spectrum per spectral bin, after masking particular spectral line regions, [OIII] for the SW spectrum and both [NII] and [CII] for the LW spectrum. Figure 3 shows the far-IR continuum maps thus obtained for the different integration ranges of wavenumbers. The figures illustrate the similarities between the distributions of the far-IR continuum emission detected in the maps at different wavenumbers, following the arch structure composed of the Radio Arc, the Arched Filaments, and their bridge. This similarity suggests that the dust temperature does not vary much over the observed region. There is close agreement between the local peaks of the SW and LW continuum maps near the Galactic center, which are located near the H5 HII region at $(\ell, b) \simeq\left(0^{\circ} .04,0^{\circ} .02\right)$ (Zhao et al. 1993; Lang et al. 2010).

We calculated the brightness ratio of the SW continuum averaged over the range $88-131 \mathrm{~cm}^{-1}$ to the LW continuum over $66-87 \mathrm{~cm}^{-1}$, the spatial distribution of which is shown in Fig. $4 \mathrm{a}$. The map notably shows small variations in the brightness ratio from $\sim 0.9$ to $\sim 1.2$, which correspond to the color temperature range from $\sim 25$ to $\sim 30 \mathrm{~K}$ by adopting a dust modified blackbody model with an emissivity power-law index of 2 . Thus, the spatial variation in the color temperature is notably small, despite the mapped area containing both active clusters and cold molecular clouds. In particular, there is no appreciable increase in the color temperature near the Arches and the Quintuplet cluster.

Figure $4 \mathrm{~b}$ shows the map of the [OIII] line emission, where the contour map of the [NII] $122 \mu \mathrm{m}$ line emission is overlaid. They are both obtained using the spectral fitting described below. 

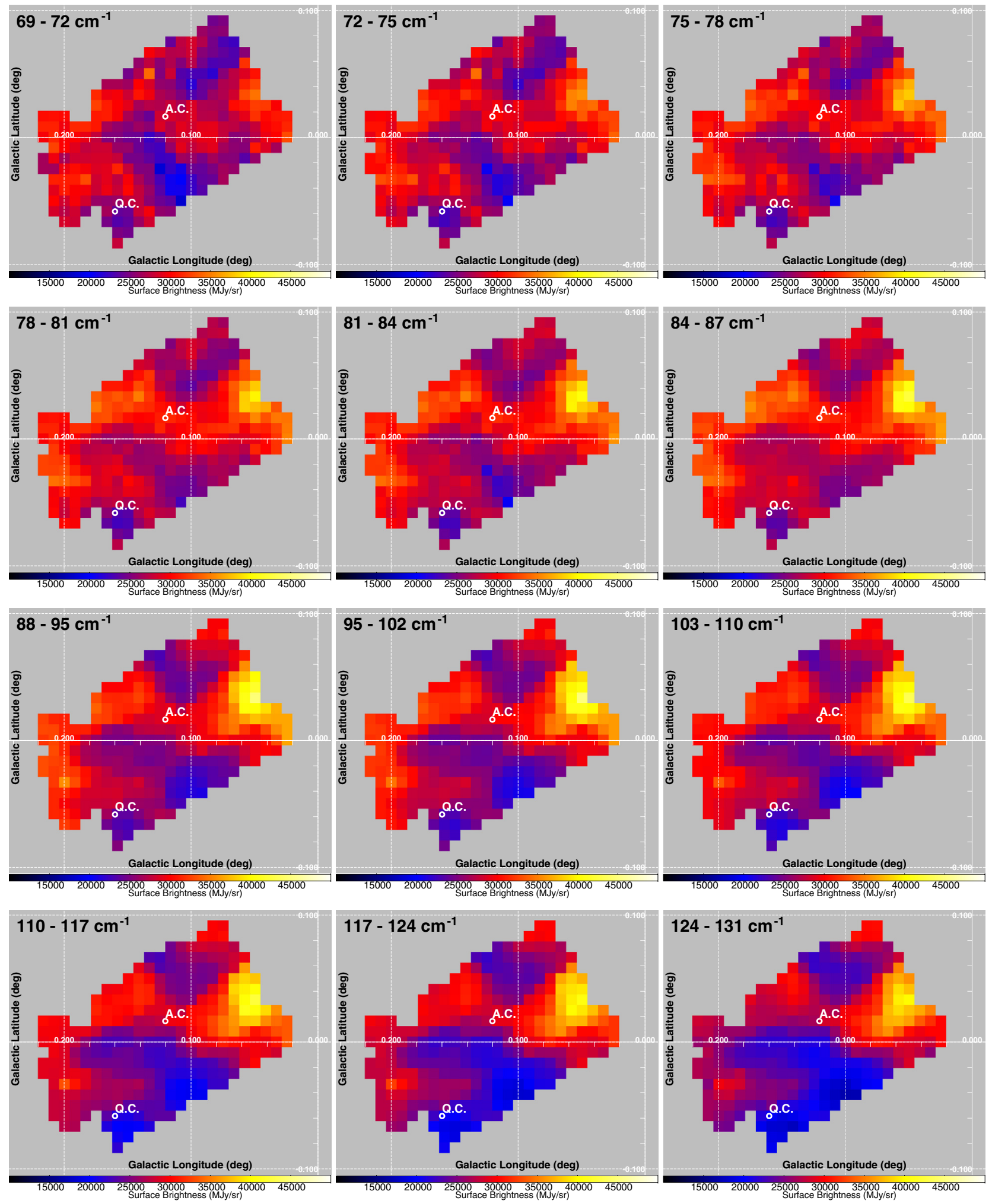

Fig. 3. Far-IR continuum maps obtained by the LW (upper six) and SW (lower six maps) arrays of AKARI FIS-FTS for the denoted integration ranges of wavenumbers. The surface brightness levels are given on common color scales among the maps.

The [OIII] line map represents the distribution of highly ionized gas, since the ionization potential of $\mathrm{O}^{+}$is as high as $35.1 \mathrm{eV}$. Judging from their spatial correspondence, the ionized gases are likely to be associated with the Arches and the Quintuplet cluster. The [NII] line emission is extended farther from the clusters than the [OIII] line emission. Since the ionization potential of $\mathrm{N}$ is $14.5 \mathrm{eV}$, Fig. $4 \mathrm{~b}$ indicates that the ionization degree of the gases heated by the two clusters decreases considerably with distance, which is consistent with the conclusions drawn by the past studies (Cotera et al. 2005; Simpson et al. 2007; Yasuda et al. 2009). Thus, in the line emission, we clearly recognize that the ISM is influenced by the two clusters, which is in marked contrast to the brightness ratio of the dust continuum emission (Fig. 4a). 
H. Kaneda et al.: Dust properties in the Galactic center

Table 1. Results of the two-temperature modified blackbody model fitting to the spectra in Figs. 2 and 8.

\begin{tabular}{lcccc}
\hline \hline Spectrum & $T_{\mathrm{w}}(\mathrm{K})$ & $T_{\mathrm{c}}(\mathrm{K})$ & $\tau_{90 \mathrm{w}}{ }^{a}$ & $\tau_{90 \mathrm{c}}{ }^{a}$ \\
\hline Figure 2a & $51 \pm 3$ & $23.0 \pm 0.3$ & $(6 \pm 1) \times 10^{-3}$ & $0.33 \pm 0.01$ \\
Figure 2c & 51 (fixed) & $21.1 \pm 0.2$ & $(6.5 \pm 0.1) \times 10^{-3}$ & $0.54 \pm 0.02$ \\
Figure 2d & 51 (fixed) & $22.7 \pm 0.2$ & $(3.8 \pm 0.1) \times 10^{-3}$ & $0.34 \pm 0.01$ \\
Figure 8 & 51 (fixed) & $19.7 \pm 0.2$ & $(5.2 \pm 0.2) \times 10^{-3}$ & $0.49 \pm 0.02,0.10 \pm 0.02^{b}$ \\
\hline
\end{tabular}

Notes. ${ }^{(a)}$ Optical depths at a wavelength of $90 \mu \mathrm{m}$ for the warm and cold components. ${ }^{(b)}$ The second cold component whose emissivity corresponds to the large graphite population with the same temperature as the first cold component (see text for details).
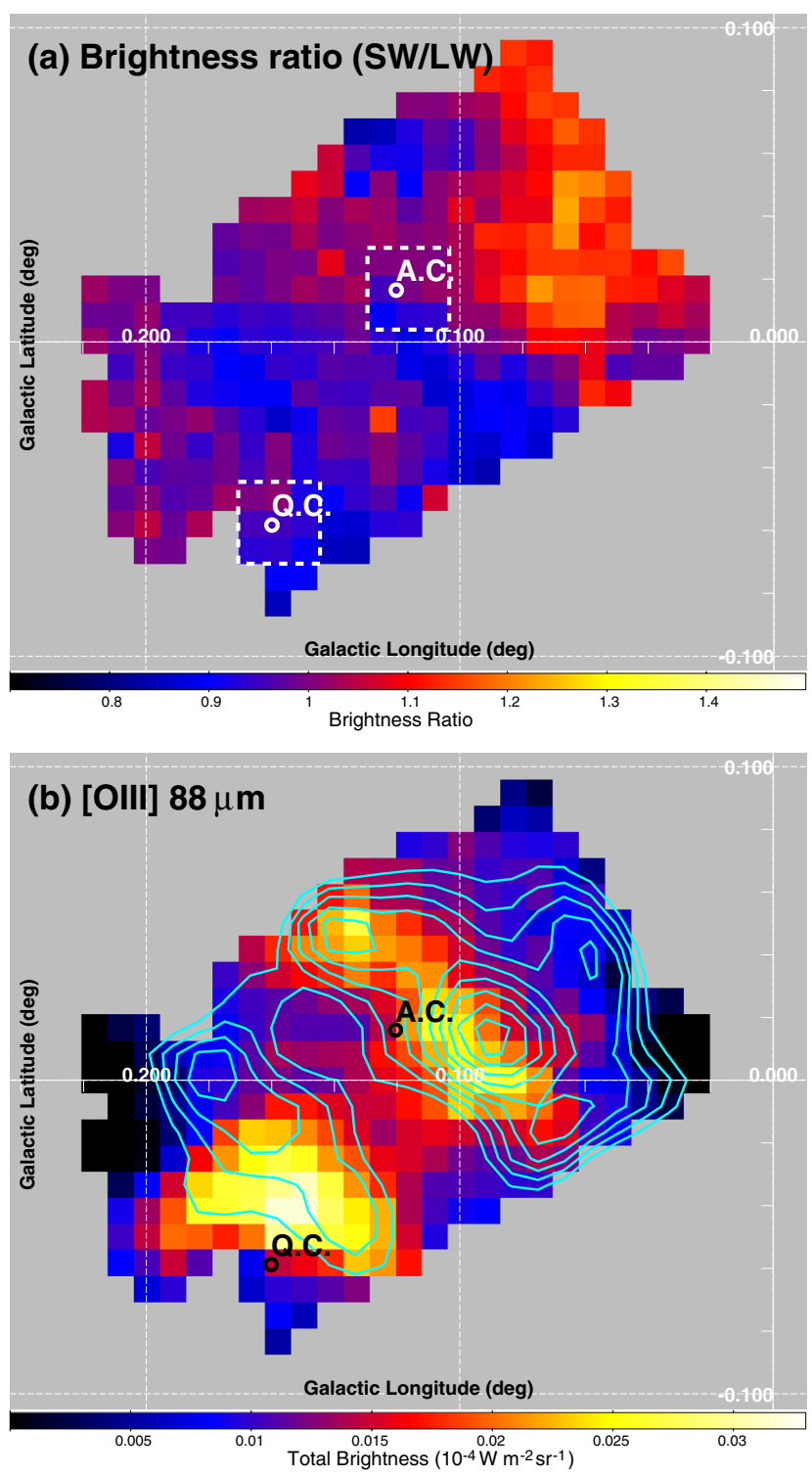

Fig. 4. a) Brightness ratio map derived from the SW continuum averaged over the range of $88-131 \mathrm{~cm}^{-1}$ that is divided by the LW continuum over $66-87 \mathrm{~cm}^{-1}$. The dashed squares indicate the aperture regions used to obtain the spectra shown in Figs. 2c and d. b) [OIII] $88 \mu \mathrm{m}$ line intensity map overlaid on the contours of the [NII] $122 \mu \mathrm{m}$ line intensity. The contour levels are linearly scaled from $4 \times 10^{-7}$ to $1.5 \times 10^{-6} \mathrm{~W} \mathrm{~m}^{-2} \mathrm{sr}^{-1}$.

\subsection{Spectra of various local areas}

Figures $2 \mathrm{c}$ and $\mathrm{d}$ show the spectra obtained for the regions toward the Arches and the Quintuplet cluster, respectively. We adopt the spectra for the $1.5 \times 1.5$ (three by three bins) apertures centered on the clusters. Most interestingly, the spectrum for the Arches cluster region appears to have a broad $\left(\sim 15 \mathrm{~cm}^{-1}\right)$ hump around $80 \mathrm{~cm}^{-1}$. In addition, the spectrum for the Arches cluster region has a slightly bluer continuum than that for the Quintuplet cluster region at large wavenumbers, which is consistent with the brightness ratio map in Fig. 4a. The [OIII] line intensities are similar between the two, while the [NII] and [CII] lines in the spectrum for the Quintuplet cluster are weaker than those in the spectrum for the Arches cluster.

To fit the entire baseline of the total spectrum in Fig. 2a, we apply a two-temperature modified blackbody model, using an emissivity power-law index $\beta=2$ (Pierce-Price et al. 2000; Rodríguez-Fernández et al. 2004) and after masking the aforementioned spectral line regions. As a result, we find that the two-temperature modified blackbody model closely reproduces the continuum shape and another temperature component is not required. It is obvious that a single-temperature model cannot reproduce the spectrum at all (Fig. 2b). The best-fit temperature of the warmer dust component is $51 \mathrm{~K}$, while that of the colder dust component is $23 \mathrm{~K}$ (Table 1 ); they roughly agree with the temperatures derived by Etxaluze et al. (2011). We then use the same model to fit the spectra of the Arches and Quintuplet clusters, adding another masking region at 80 to $90 \mathrm{~cm}^{-1}$ because of the apparent presence of the broad hump over these wavenumbers. Since the temperature of the warm dust component is poorly constrained, we fix its temperature to $51 \mathrm{~K}$, which is the best-fit value for the total spectrum. As a result, Fig. 2 clearly shows that the spectrum toward the Arches cluster has a broad excess component on top of the dust modified blackbody continuum, which is too narrow to be explained by another modified blackbody component. In contrast, the spectrum toward the Quintuplet cluster does not exhibit this excess component.

Figure 5 displays examples of spectra derived for the local areas of $30^{\prime \prime} \times 30^{\prime \prime}$. Their spatial variation along the line connecting the Arches and Quintuplet clusters is shown in the upper panels, while that along the Galactic plane is presented in the lower panels. The shape of the spectrum varies significantly from area to area. In particular, the spectra of the regions around $(\ell, b) \simeq\left(0^{\circ} .13,0^{\circ} .0\right)$ exhibit a relatively strong hump around $80-90 \mathrm{~cm}^{-1}(110-130 \mu \mathrm{m})$, as seen in the spectrum of the Arches cluster (Fig. 2c). Since some local spectra show unrealistically steep continuum slopes at wavenumbers smaller than $64 \mathrm{~cm}^{-1}$ $(>156 \mu \mathrm{m})$, probably due to the calibration errors around the cuton wavenumber of the detector responsivity that varies among the array pixels (Murakami et al. 2010), we additionally mask the spectral region at $<65 \mathrm{~cm}^{-1}$. We fit all the local spectra with the two dust modified blackbody components adopted in the above fitting, plus the [OIII] and [NII] emission lines, the $\mathrm{OH}$ absorption line, and the excess component. All of these line components are assumed to be narrow lines, while the excess component is assumed to have a Lorentzian profile with a center 

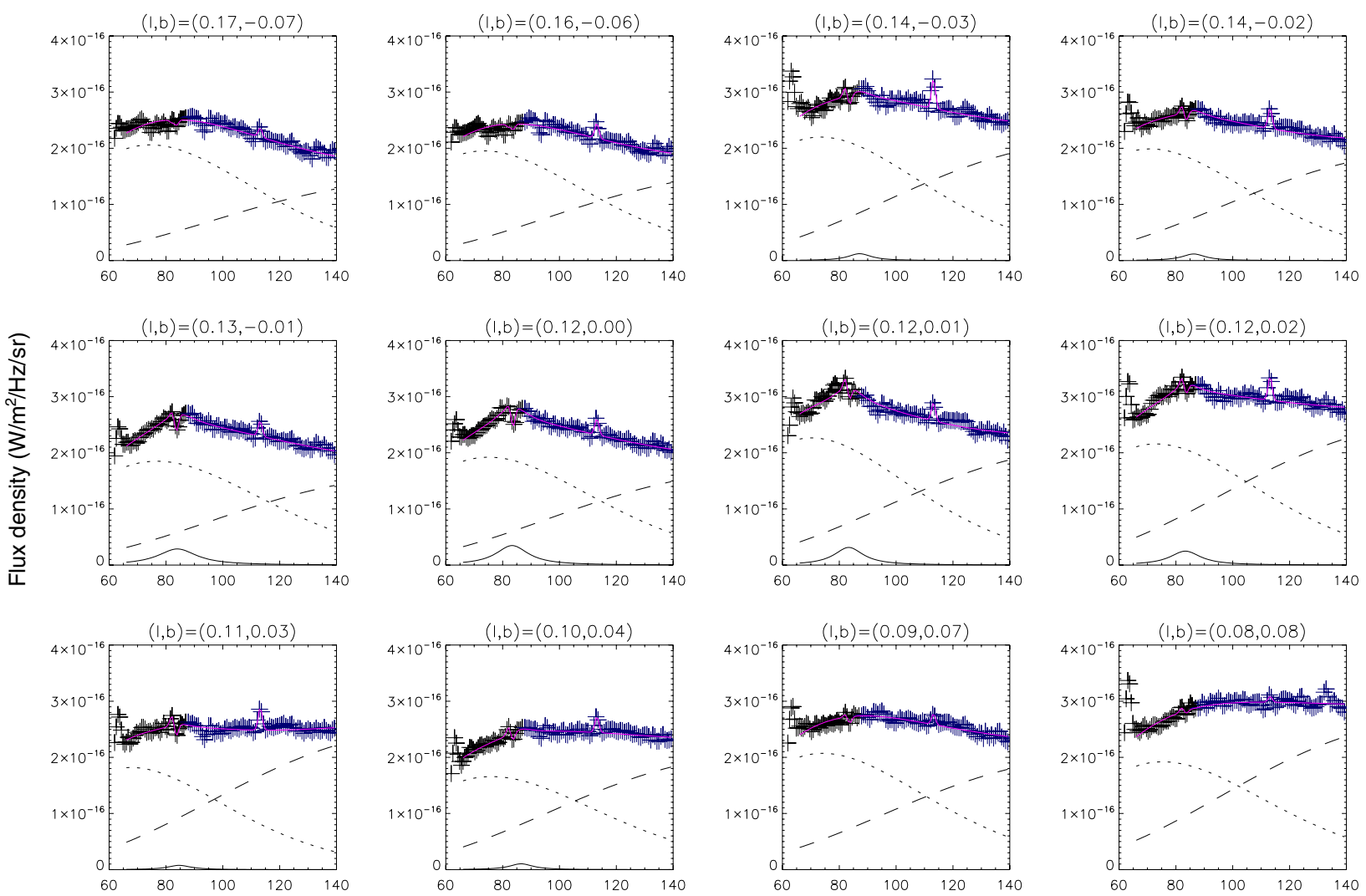

Wavenumber $(/ \mathrm{cm})$
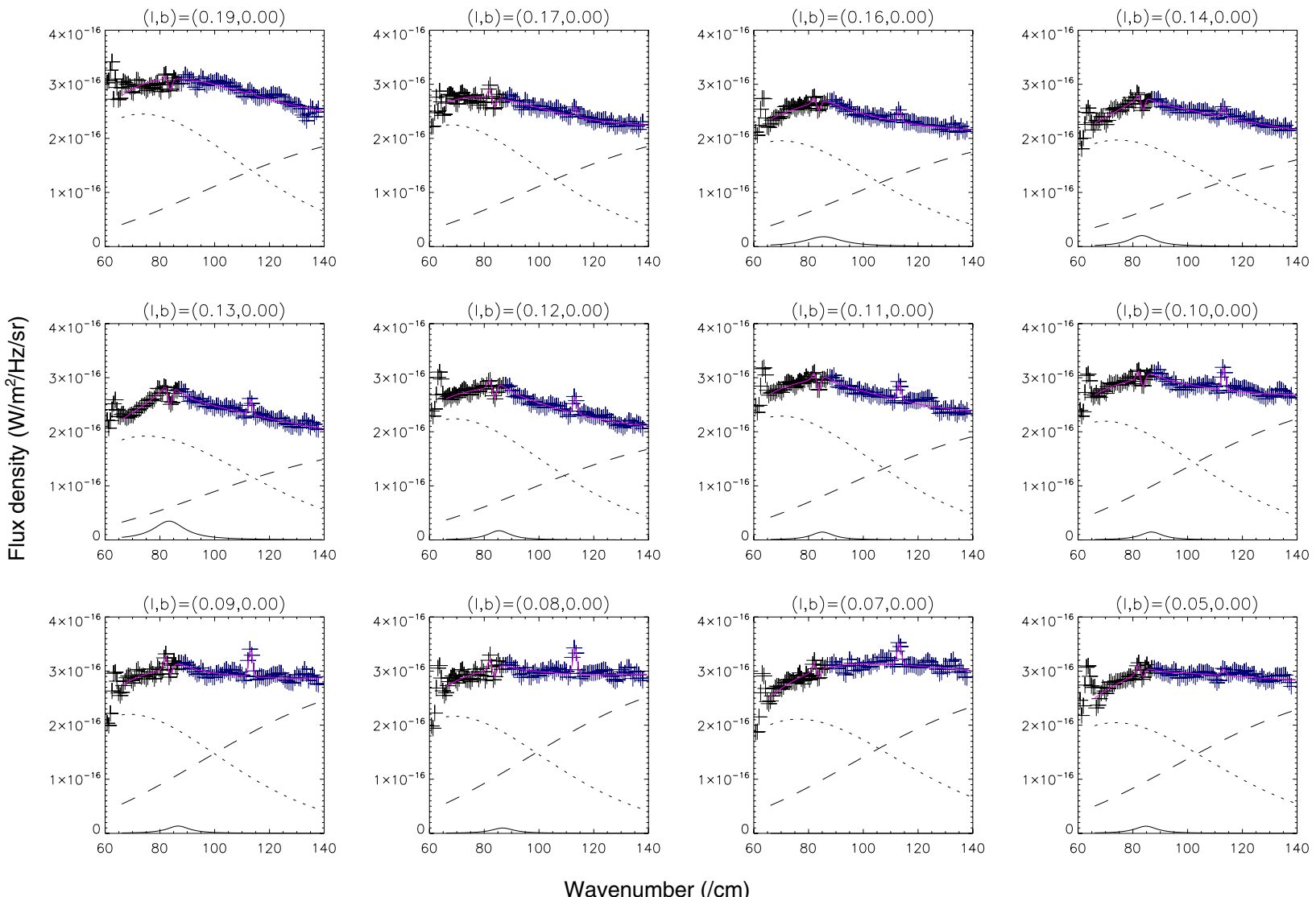

Wavenumber $(/ \mathrm{cm})$

Fig. 5. Spectra of local $\left(30^{\prime \prime} \times 30^{\prime \prime}\right)$ areas. The upper panels show the variation along the line connecting the Arches and Quintuplet clusters, while the lower panels show that along the Galactic plane. For each spectrum, its position is denoted at the top in Galactic coordinates. The solid curves indicate the best-fit model that includes two dust modified blackbody components, the [OIII] and [NII] emission lines, the $\mathrm{OH}$ absorption line, and the excess component represented by a Lorentzian function (see text for details). 

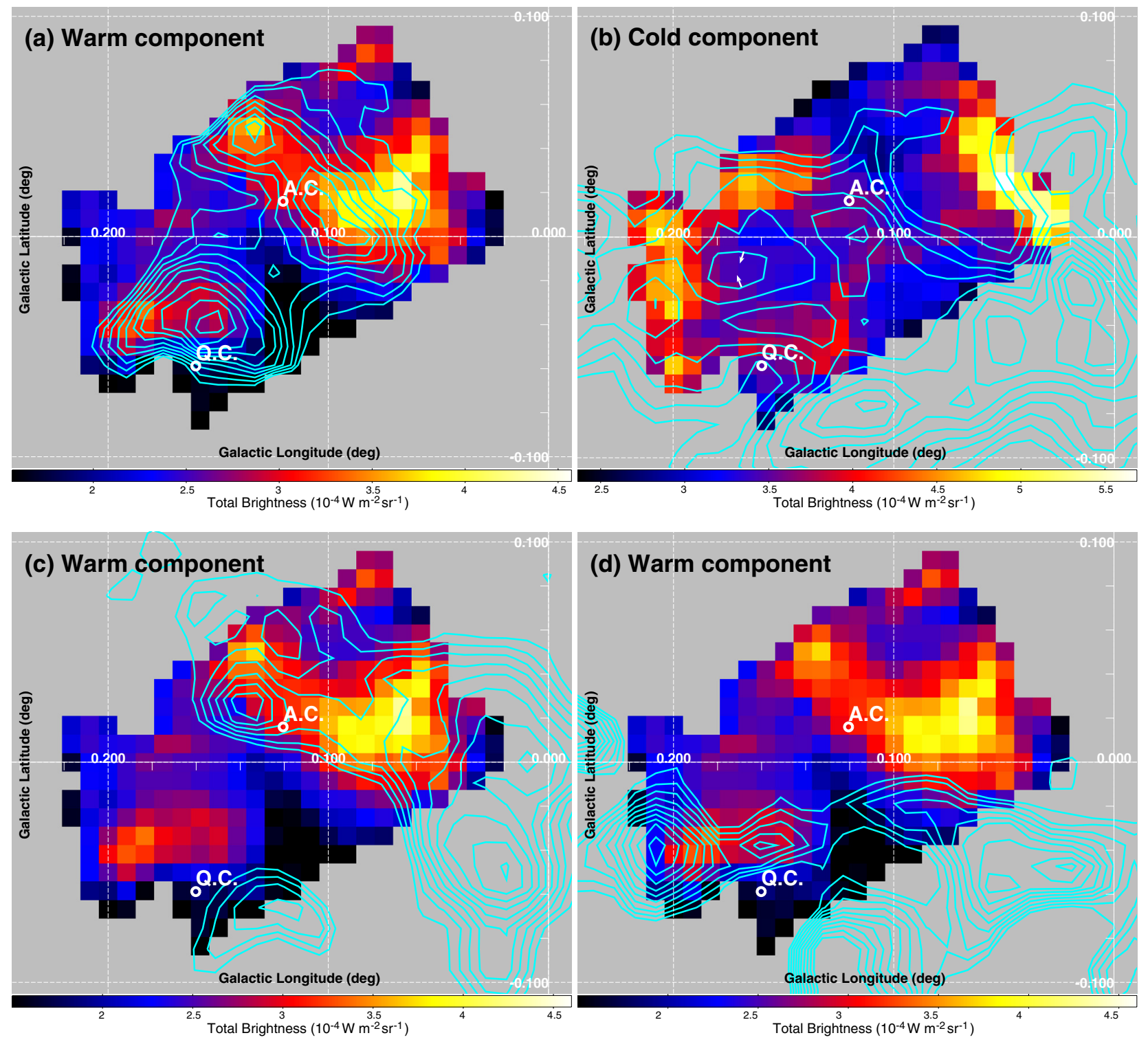

Fig. 6. a) Distribution of the total brightness of the warm dust component with the contour map of the [OIII] line emission from the same data as in Fig. 4b. The contour levels are linearly drawn from $1 \times 10^{-6}$ to $3 \times 10^{-6} \mathrm{~W} \mathrm{~m}^{-2} \mathrm{sr}^{-1}$. b) Distribution of the total brightness of the cold dust component with the contour map of the ${ }^{12} \mathrm{CO} J=1-0$ emission integrated over the total velocity range $\left(V_{\mathrm{LSR}}=-220\right.$ to $+220 \mathrm{~km} \mathrm{~s}^{-1}$; Oka et al. 1998). The contours are linearly drawn from 690 to $1200 \mathrm{~K} \mathrm{~km} \mathrm{~s}^{-1}$. c) The contour maps of the ${ }^{12} \mathrm{CO} J=1-0$ emission integrated over the velocity range of -30 to $-10 \mathrm{~km} \mathrm{~s}^{-1}$ and d) that over +20 to $+40 \mathrm{~km} \mathrm{~s}^{-1}$, both superimposed on the same distribution map of the warm dust component as shown in panel a). The contours are linearly drawn from 50 to $130 \mathrm{~K} \mathrm{~km} \mathrm{~s}^{-1}$ for panel c) and from 160 to $240 \mathrm{~K} \mathrm{~km} \mathrm{~s}^{-1}$ for panel d).

that varies between 80 and $95 \mathrm{~cm}^{-1}$ and a width between 10 and $20 \mathrm{~cm}^{-1}$. All the spectra were fairly closely reproduced by the model; examples of the fitting results can be seen in Fig. 5.

As a result, a local spectrum is decomposed into the three continuum components as well as the three line components at every spatial bin, and their spatial distributions are then obtained. Each component is integrated with respect to the wavenumber; the total brightnesses of the warm and cold dust components are obtained by integrating over the observed wavenumber range of $60-140 \mathrm{~cm}^{-1}$. The distributions of the warm and cold dust components are shown in Figs. 6a and b, while the distribution of the excess component is shown in Fig. 7a. The results for the [OIII] and [NII] lines are used in Fig. 4b. The $\mathrm{OH}$ absorption line flux is divided by the continuum intensity at the central wavenumber to derive its equivalent width, the distribution of which is shown in Fig. 7b. The $\mathrm{OH}$ absorption due to the fundamental rotational mode becomes quite easily optically thick (Goicoechea et al. 2002), hence it can become saturated in high-resolution spectra of relatively strong regions, which would then give us only qualitative information.

We find that the warmer dust emission is spatially correlated with the [OIII] line emission (Fig. 6a) and also with the [NII] line emission, which is extended farther from the two clusters (Fig. 4b). As can be seen in Fig. 7, the distribution of the excess emission, which appears to differ from that of the warm dust emission, is notably similar to that of the $\mathrm{OH}$ absorption equivalent width. This spatial correlation, as well as their likely CO cloud counterpart shown below, convinces us that the excess is not an artifact but a real spectral feature. In addition, the two independent detectors (i.e., SW and LW), which both cover the excess component, consistently show an excess in the spectra. 

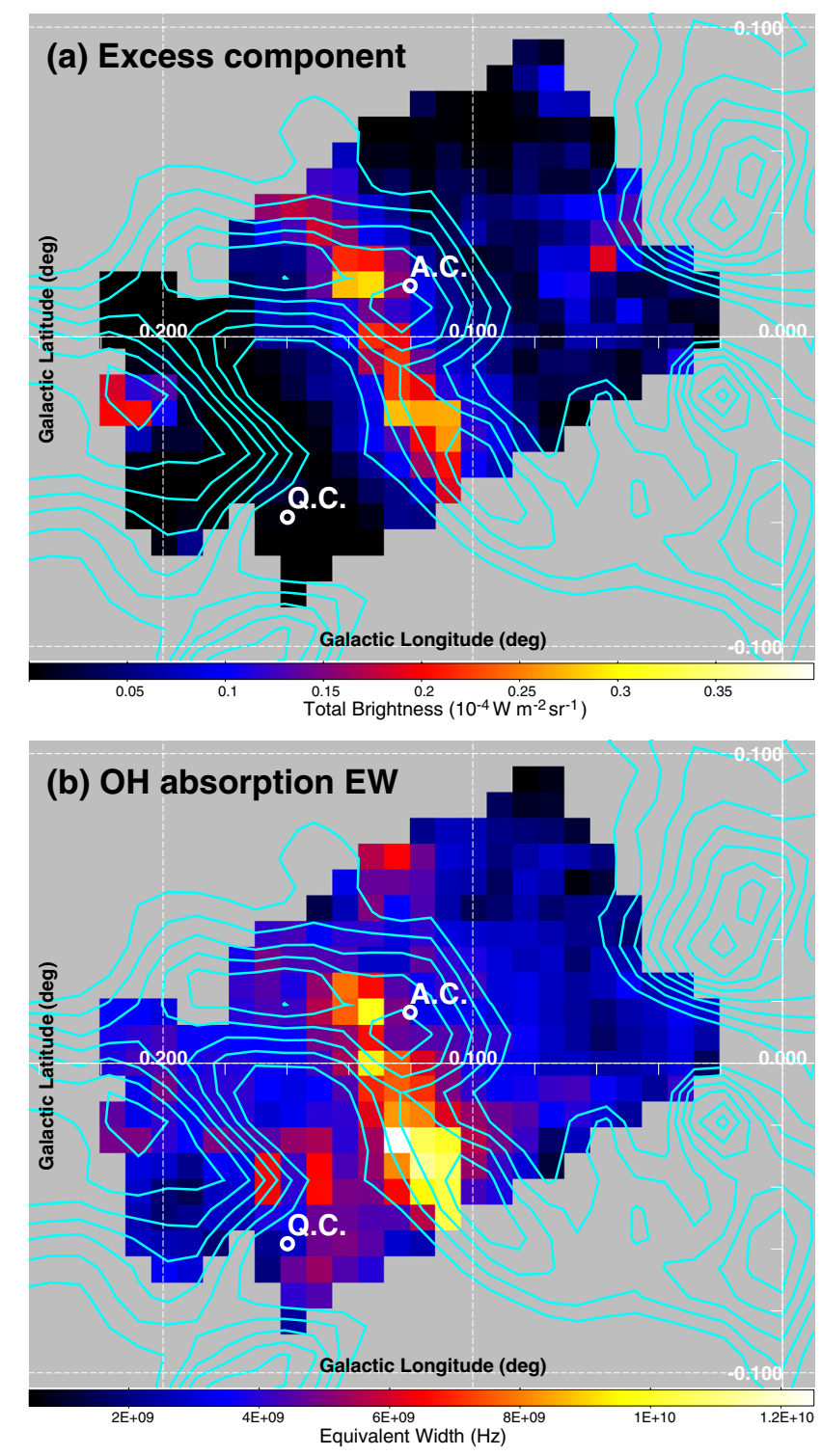

Fig. 7. a) Distribution of the total brightness of the excess component on the contour map of the ${ }^{12} \mathrm{CO}(J=1-0)$ integrated over the velocity range of +70 to $+90 \mathrm{~km} \mathrm{~s}^{-1}$. The contours are linearly drawn from 100 to $280 \mathrm{~K} \mathrm{~km} \mathrm{~s}^{-1}$. b) Distribution of the equivalent width of the $\mathrm{OH} 119 \mu \mathrm{m}$ absorption line, which is derived by dividing the line flux by the continuum intensity at the central wavenumber. The contour map is the same as that in panel a).

\section{Discussion}

\subsection{Warm dust component and its relation to the molecular clouds}

As shown in Fig. 6a, the warm dust emission derived from the spectral decomposition is likely to be associated with the clouds influenced by the Arches and Quintuplet clusters. It is clear from the figure that the warm dust is more abundant near the Arches cluster than near the Quintuplet cluster. Using the optical depths $\tau_{v}$ derived from the spectral fitting, we estimated the mass of the warm dust existing around each cluster. For the square apertures of $1.5 \times 1.5$ used to create the spectra in Figs. $2 \mathrm{c}$ and d, for the Arches cluster $\tau_{v}$ at $90 \mu \mathrm{m}$ is $6.5 \times 10^{-3}$ and for the Quintuplet cluster it is $3.8 \times 10^{-3}$ (Table 1). Using the equation $M_{\text {dust }}=\tau_{v} A / \kappa_{v}$, where $A$ is the physical area $\sim 2 \times 10^{38} \mathrm{~cm}^{2}$ for a distance to the Galactic center of $8.5 \mathrm{kpc}$ and $\kappa_{v}$ is the mass absorption coefficient of $25 \mathrm{~cm}^{2} \mathrm{~g}^{-1}$ at $90 \mu \mathrm{m}$ (Dorschner et al. 1995), we obtain the warm dust masses of $26 M_{\odot}$ and $15 M_{\odot}$ for the Arches and the Quintuplet cluster, respectively. For comparison, we estimate the cold dust masses to be $2.1 \times 10^{3} M_{\odot}$ and $1.3 \times 10^{3} M_{\odot}$ with the same apertures for the Arches and the Quintuplet cluster, respectively. Since our far-IR coverage is insensitive to the presence of even colder dust, they are rather lower limits to the dust masses. Hence, the warm dust makes only a negligible contribution to the total dust mass even toward the two active clusters, causing the clusters to be almost invisible in the far-IR dust continuum emission maps (Fig. 3) and the brightness ratio map (Fig. 4a).

The warm dust clouds are distributed anistropically around the Arches and the Quintuplet cluster, and probably trace the highly-ionized gas locally heated by intense stellar radiation from the two clusters. We then search for corresponding molecular clouds based on the ${ }^{12} \mathrm{CO}(J=1-0)$ map (Oka et al. 1998). As can be seen in Figs. $6 \mathrm{c}$ and d, we find that the $\mathrm{CO}$ clouds with velocities of $V_{\mathrm{LSR}}=-30$ to $-10 \mathrm{~km} \mathrm{~s}^{-1}$ and +20 to $+40 \mathrm{~km} \mathrm{~s}^{-1}$ are spatially correlated with the warm dust distributions near the Arches and the Quintuplet cluster, respectively. It is therefore obvious that the warm dust clouds containing the two clusters have different radial velocities. The velocity ranges are consistent with the velocities of $\sim-30 \mathrm{~km} \mathrm{~s}^{-1}$ and $\sim+40 \mathrm{~km} \mathrm{~s}^{-1}$ (Serabyn \& Güsten 1987, 1991; Lang et al. 2010) measured for the Arched Filaments and the Sickle HII region, which are thought to be ionized by the Arches and the Quintuplet cluster, respectively. In other words, our results strongly imply that they are related.

A larger amount of warm dust exists around the Arches cluster, while the [OIII] emission is more prominent near the Quintuplet cluster. Thus, there are differences between the properties of the ISM around the two clusters, presumably reflecting that the clusters differ in age; the Arches cluster is relatively young (2.0-2.5 Myr; Figer et al. 2002; Najarro et al. 2004) retaining a larger amount of the surrounding ISM, while the Quintuplet cluster is more evolved ( 4 Myr; Figer et al. 1999) containing a variety of massive stars such as luminous blue variables, one of which is the well-known Pistol star.

In contrast, the cold dust component does not show any spatial correspondence with either the Arches or the Quintuplet cluster. In Fig. 6b, we compare the distribution of the cold dust with that of the $\mathrm{CO}$ emission integrated over the total velocity range $\left(V_{\mathrm{LSR}}=-220\right.$ to $\left.+220 \mathrm{~km} \mathrm{~s}^{-1}\right)$. The figure shows that they have spatial distributions similar to each other. This is reasonable because the cold dust emission represents a significant fraction of the total gas amount integrated along the line of sight. We find that most of the cold dust is unlikely to be associated with the two clusters.

\subsection{Excess component over dust modified blackbody emission}

We find that there is a significant excess of emission on top of a modified blackbody continuum around $80-90 \mathrm{~cm}^{-1}$ (110-130 $\mu \mathrm{m})$, which probably represents a dust feature. From the spatial information, it is clear that this spectral component is not associated with either of the two clusters. By comparison with the CO map, a molecular cloud with velocities of +70 to $+90 \mathrm{~km} \mathrm{~s}^{-1}$ is found to exhibit a good spatial correspondence to the excess component. Judging from both this velocity range and spatial position, the cloud seems to be part of the 100-pc ring revealed by Herschel around the Galactic center (Molinari et al. 2011) and to reside in the foreground of the 
Arches cluster, which appears to be located on the back side of the ring $\left(-30\right.$ to $\left.-10 \mathrm{~km} \mathrm{~s}^{-1}\right)$. From the negative to the positive longitude side of the Galactic center, the $+20 \mathrm{~km} \mathrm{~s}^{-1}$ cloud, the $+50 \mathrm{~km} \mathrm{~s}^{-1}$ cloud, and the above-mentioned +70-+90 $\mathrm{km} \mathrm{s}^{-1}$ cloud are found next to each other, possibly forming part of the front side of the 100-pc ring. The position of Sgr A* is probably shifted from the center of the ring toward the front side (Molinari et al. 2011), which can be so close that the cloud may be significantly influenced by Sgr A*.

With ISO, Kemper et al. (2002) detected a dust feature at $93 \mu \mathrm{m}$ from the dust shells of evolved stars, which was identified as calcite $\left(\mathrm{CaCO}_{3}\right)$. Although the excess emission we detect appears in a similar far-IR region, it is a little shifted toward longer wavelengths $(110-130 \mu \mathrm{m})$ and is also certainly of interstellar origin. Hence, among likely interstellar grains, we suggest that the excess component may represent a graphite dust feature. Onaka \& Okada (2003) reported the detection of a similar broad feature at wavelengths from $80 \mu \mathrm{m}$ to $140 \mu \mathrm{m}$ in the ISO/LWS spectra of the diffuse emission from the Carinae Nabula and Sharpless 171 . They pointed out that the feature may originate from carbon onion grains. Graphite has an interband transition around $80 \mu \mathrm{m}$ in the direction parallel to the graphitic plane (Philipp 1977). However, the interband transition is hardly visible for the ensemble of randomly oriented graphite spheres, because the absorption efficiency for the electric field parallel to the graphitic plane is much lower than that for the electric field vertical to the plane (Draine \& Lee 1984). In the carbon onions, the graphitic planes are curved, forming closed shells, and the optical properties in the direction parallel to the graphic plane interact with those in the perpendicular direction, which causes the interband feature to become visible in the emissivity (Henrard et al. 1993). The position and profile of the interband feature are expected to depend significantly on the assumed optical constants, especially the behavior of free electrons that depends on the temperature and the localization of $\pi$ electrons (Tomita et al. 2001). Therefore, the peak position of the feature can be shifted to wavelengths longer than $100 \mu \mathrm{m}$ (Onaka \& Okada 2003). Since the harsh conditions in which interstellar processes occur appear to favor the formation of onions (Ugarte 1995), our detection of a feature from the Galactic center that is similar to that in the active star forming regions studied by Onaka \& Okada (2003) seems to be plausible.

As an alternative, we propose that large graphite grains may dominate the far-IR emissivity. In the case of graphite grains larger than $0.5 \mu \mathrm{m}$, the magnetic dipole absorption due to energy losses produced by the eddy currents in the graphitic plane becomes substantial, and can have a wavenumber dependence that reproduces a spectral feature similar to the observed one (Draine $\&$ Lee 1984). Using the absorption efficiency $Q_{\mathrm{abs}}(a, \lambda)$ tabulated by Draine (Laor \& Draine 1993) for the dust temperature of $25 \mathrm{~K}$, where $a$ is the grain size, we calculated the total emissivity for two kinds of the size distributions for graphite dust: $n(a) \mathrm{d} a \propto a^{-3.5} \mathrm{~d} a$ from $a_{\min }=0.001 \mu \mathrm{m}$ to $a_{\max }=0.25 \mu \mathrm{m}$, and $n(a) \mathrm{d} a \propto a^{-2.5} \mathrm{~d} a$ from $a_{\min }=0.001 \mu \mathrm{m}$ to $a_{\max }=4 \mu \mathrm{m}$. The former is a standard distribution (Mathis et al. 1977), while the latter is the distribution strongly biased toward larger sizes. We then fit the spectrum in Fig. 2c by a dust model consisting of two cold and one warm dust components, where the second cold component has the emissivity of the large graphite population, while the others have the emissivity of a standard grain-size population. For simplicity, we assume that the two cold components have the same temperature but that the fraction of the second to the first cold component and their common temperature are allowed to vary, while the temperature of the warm dust component is

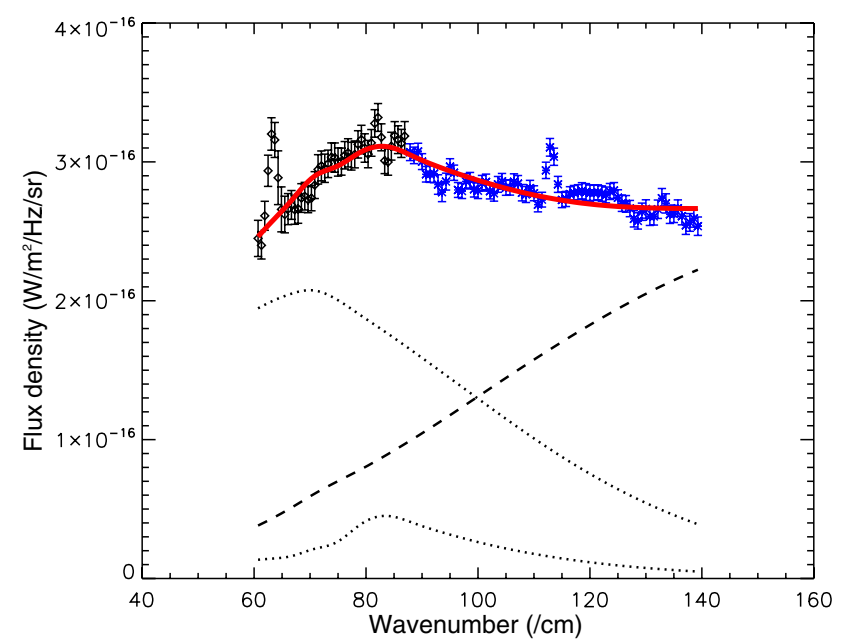

Fig. 8. Same as Fig. 2c, but the spectrum is fitted by the warm and cold dust model including the second cold component with the large graphite population (see text for details). The two cold components and the warm component are indicated by the dotted lines and the dashed line, respectively.

fixed to $51 \mathrm{~K}$ as above. As a result, the dust model including the large graphite grains reproduces the observed spectrum fairly well (Fig. 8), where the best-fit temperature of the cold dust components is $\sim 20 \mathrm{~K}$ (Table 1 ). In terms of the dust mass, the additional large-grain component contributes to about $5 \%$ of the total. Pagani et al. (2010) discovered the ubiquity of micron-sized dust grains in the dense ISM, using a so-called core-shine effect. Therefore, it seems that grains much larger than standard dust grains can be present, but they must be mostly composed of graphite.

It is also intriguing that the $\mathrm{OH}$ absorption is particularly enhanced in the region bright in the excess emission, as seen in Fig. 7. The $\mathrm{OH}$ absorption and the excess emission are thus likely to originate in the same $+70-+90 \mathrm{~km} \mathrm{~s}^{-1}$ cloud. The enhanced $\mathrm{OH}$ abundances are predicted in either molecular shock regions (Draine et al. 1983) or the outer layers of photo-dissociation regions (PDRs, Sternberg \& Dalgarno 1995; Goicoechea et al. 2004). In the case of dust shattering, the molecular shock would be hostile to the survival of the large grains, hence the PDR picture be more favorable. Both interpretations for the dust feature indicate that it corresponds to grains of graphite, although graphite materials are no longer believed to be major components of interstellar carbonaceous grains, amorphous carbon materials being thought to be more likely (e.g., Compiégne et al. 2011). The detection of the excess feature from this region might be related to the (past) activity of Sgr A*, because intense UV heating is needed to graphitize carbonaceous grains.

\section{Summary}

We have presented the results of a far-IR FTS $\left(60-140 \mathrm{~cm}^{-1}\right.$; $R=1.2 \mathrm{~cm}^{-1}$ ) spectral mapping of the Galactic center region of an area of about $10^{\prime} \times 10^{\prime}$, to investigate the properties of interstellar dust around the massive star-forming clusters, Arches and Quintuplet. The maps of the continuum emission reveal similar spatial distributions at a range of wavenumbers $\left(69-131 \mathrm{~cm}^{-1}\right)$, which do not clearly show any spatial correspondence to either the Arches or the Quintuplet cluster. There is no appreciable increase in the color temperature of the dust emission near 
the Arches and Quintuplet clusters, whereas strong [OIII] line emission is clearly associated with the two clusters. Two modified blackbody components with different temperatures $(\sim 50 \mathrm{~K}$ and $\sim 20 \mathrm{~K}$ ) can closely reproduce the continuum shapes of most of the local spectra. Some spectra, however, contain a significant excess on top of a modified blackbody continuum around $80-90 \mathrm{~cm}^{-1}(110-130 \mu \mathrm{m})$, probably representing a dust feature. We have found that the spatial distribution of the warmer dust component is strongly correlated with that of the [OIII] line emission, hence that the warm dust is likely to be associated with the highly ionized gas locally heated by intense stellar radiation from the two clusters. The excess component has an apparently different distribution from that of the warm dust component.

We have found that the $\mathrm{CO}$ clouds with different velocity ranges of -30 to $-10,+20$ to +40 , and +70 to $+90 \mathrm{~km} \mathrm{~s}^{-1}$ are spatially correlated, respectively, with the warm dust component around the Arches cluster, that around the Quintuplet cluster, and the excess component. The first two velocity ranges agree with those measured for the Arched Filaments and the Sickle HII region that are thought to be associated with the Arches and the Quintuplet cluster, respectively. We therefore confirm the connections between the clusters and the HII regions, and successfully resolve the parts of the CO clouds heated by the Arches and Quintuplet clusters. The last velocity range suggests that the cloud associated with the excess component resides in the foreground of the Arches cluster. Together with the $+20 /+50 \mathrm{~km} \mathrm{~s}^{-1}$ clouds, it appears to form the front part of the 100-pc ring in the vicinity of Sgr A*, where some grains might be highly processed to emit the far-IR feature. We discuss possible origins of the dust feature based on graphite materials, namely carbon onions and micron-sized graphite grains. We find that the dust model including the large graphite grains can at least reproduce the observed spectrum with the dust feature fairly well.

Acknowledgements. We thank all the members of the AKARI project. AKARI is a JAXA project with the participation of ESA. We use the archival ${ }^{12} \mathrm{CO}$ $(J=1-0)$ survey map of the Galactic center provided by Oka et al. (1998). This research is supported by a Grant-in-Aid for Scientific Research No. 22340043 from the Japan Society for the Promotion of Science, and the Nagoya University Global COE Program, "Quest for Fundamental Principles in the Universe: from Particles to the Solar System and the Cosmos", from the Ministry of Education, Culture, Sports, Science and Technology of Japan.

\section{References}

Compiégne, M., Verstraete, L., Jones, A., et al. 2011, A\&A, 525, A103 Cotera, A. S., Erickson, E. F., Simpson, J. P., et al. 1992, BAAS, 24, 1262 Cotera, A. S., Erickson, E. F., Colgan, S. W. J., et al. 1996, ApJ, 461, 750
Cotera, A. S., Colgan, S. W. J., Simpson, J. P., \& Rubin, R. H. 2005, ApJ, 622, 333

Cox, P., \& Laureijis, R. 1989, in The Center of the Galaxy, ed. M. Morris, IAU Symp., 136, 121

Dorschner, J., Begemann, B., Henning, T., Jäger, C., \& Mutschke, H. 1995 A\&A, 300, 503

Draine, B. T., \& Lee, H. M. 1984, ApJ, 285, 89

Draine, B. T., Roberge, W. G., \& Dalgarno, A. 1983, ApJ, 264, 485

Etxaluze, M., Smith, H. A., Tolls, V., Stark, A. A., \& González-Alfonso, E. 2011, AJ, 142,134

Figer, D. F., McLean, I. S., \& Morris, M. 1999, ApJ, 514, 202

Figer, D. F., Najarro, F., Gilmore, D., et al. 2002, ApJ, 581, 258

Genzel, R., Stacey, G. J., Harris, A. I., et al. 1990, ApJ, 356,160

Goicoechea, J. R., \& Cernicharo, J. 2002, ApJ, 576, L77

Goicoechea, J. R., Rodríguez-Fernández, N. J., \& Cernicharo, J. 2004, ApJ, 600, 214

Henrard, L., Lucas, A. A., \& Lambin, Ph. 1993, ApJ, 406, 92

Kawada, M., Baba, H., Barthel, P. D., et al. 2007, PASJ, 59, 389

Kawada, M., Takahashi, H., Murakami, N., et al. 2008, PASJ, 60, 389

Kemper, F., Jäger, C., Waters, L. B. F. M., et al. 2002, Nature, 415, 295

Lang, C. C., Goss, W. M., \& Morris, M. 2001, AJ, 121, 2681

Lang, C. C., Johnson, K. E., Goss, W. M., \& Rodríguez, L. F. 2005, AJ, 130, 2185

Lang, C. C., Goss, W. M., Cyganowski, C., \& Clubb, K. I. 2010, ApJS, 191, 275

Laor, A., \& Draine, B. T. 1993, ApJ, 402, 441

Lis, D. C., Serabyn, E., Zylka, Z., \& Li, Y., 2001, 550, 761

Mathis, J. S., Rumpl, W., \& Nordsieck, K. H. 1977, ApJ, 217, 425

Molinari, S., Bally, J., Noriega-Crespo, A., et al. 2011, ApJ, 735, L33

Murakami, H., Baba, H., Barthel, P., et al. 2007, PASJ, 59, 369

Murakami, N., Kawada, M., Ootsubo, T., et al. 2010, PASJ, 62, 1155

Nagata, T., Woodward, C. E., Shure, M., \& Kobayashi, N. 1995, AJ, 109,1676

Najarro, F., Figer, D. F., Hillier, D. J., \& Kudritzki, R. P. 2004, ApJ, 611, L105

Nakagawa, T., Yui, Y. Y., Doi, Y., et al. 1998, ApJS, 115, 259

Onaka, T., \& Okada, Y. 2003, ApJ, 585, 872

Pagani, L., Steinacker, J., Bacmann, A., Stutz, A., \& Henning, T. 2010, Science, 329, 1622

Pierce-Price, D., Richer, J. S., Greaves, J. S., et al. 2000, ApJ, 545, L121

Philipp, H. R. 1977, Phys. Rev. B, 16, 2896

Oka, T., Hasegawa, T., Sato, F., Tsuboi, M., \& Miyazaki, A. 1998, ApJS, 118, 455

Rodríguez-Fernández, N. J., Martín-Pintado, J., \& de Vicente, P. 2001, A\&A, 377, 631

Rodríguez-Fernández, N. J., Martín-Pintado, J., Fuente, A., \& Wilson, T. L. 2004, A\&A, 427, 217

Serabyn, E., \& Güsten, R. 1987, A\&A, 184, 133

Serabyn, E., \& Güsten, R. 1991, A\&A, 242, 376

Serabyn, E., Shupe, D., \& Figer, D. F. 1998, Nature, 394, 448

Simpson, J. P., Colgan, S. W. J., Cotera, A. S., et al. 1997, ApJ, 487, 689

Simpson, J. P., Colgan, S. W. J., Cotera, A. S., et al. 2007, ApJ, 670, 1115

Sternberg, A., \& Dalgarno, A. 1995, ApJS, 99, 565

Tomita, S., Sakurai, M., Ohta, H., \& Hayashi, S. 2001, J. Chem. Phys., 114, 7477

Ugarte, D. 1995, ApJ, 443, L85

Yasuda, A., Nakagawa, T., Spaans, M., Okada, Y., \& Kaneda, H. 2008, A\&A, 480, 157

Yasuda, A., Kaneda, H., Nakagawa, T., et al. 2009, PASJ, 61, 511

Yusef-Zadeh, F., Morris, M., \& Chance, D. 1984, Nature, 310, 557

Yusef-Zadeh, F., Hewitt, J. W., Arendt, R. G., et al. 2009, ApJ, 702, 178

Zhao, J.-H., Desai, K., Goss, W. M., \& Yusef-Zadeh, F. 1993, ApJ, 418, 235 